\section{Physician burnout: let's avoid unsubstantiated claims}

\author{
Danijela Mirkovic and Renzo Bianchi
}

\begin{abstract}
Murali and Banerjee recently addressed the issue of 'burnout' in oncologists
\end{abstract} (Let's address burnout in oncologists and reimagine the way we work. Nat. Rev. Clin. Oncol. https://doi.org/10.1038/s41571-0180097-9 (2018) $)^{1}$. In their Comment article, these authors reported on the prevalence of, risk factors for and occupational outcomes associated with physician burnout. We salute the authors' efforts to provide the reader with an overview of burnout among oncologists and we recognize the importance of combatting chronic occupational stress in this profession; however, we argue that the authors' article does not adequately articulate the limitations of research into physician burnout and overlooks important findings pertaining to this area of research.

First, Murali and Banerjee indicated that a "substantial proportion of physicians" suffers from burnout. More specifically, the authors mentioned a $71 \%$ mean prevalence of burnout among European oncologists. Problematically, the authors' claims are based on findings that are clinically and nosologically foundationless ${ }^{2}$. Currently, burnout is not a nosological category and no (differential) diagnosis for burnout exists ${ }^{2,3}$. No consensus exists, for instance, on the symptoms that should be considered in clinical assessments of burnout; the minimal required severity, duration and frequency of these symptoms; or the expected effects of the exhibited symptoms on the patient's (work) life. Because what constitutes a case of burnout is undefined, the prevalence of the syndrome cannot be established. As highlighted by many investigators, the estimates of the prevalence of burnout reported in the research literature involved the use of arbitrary cut-off scores that were not designed for diagnostic purposes ${ }^{2-4}$. Burnout has formally been described as a syndrome combining a 'high' level of emotional exhaustion, a 'high' level of depersonalization and a 'low' sense of personal accomplishment; however, what 'high' and 'low' mean in this formal description remains elusive. Moreover, in many studies of the prevalence of burnout, participants were categorized as 'burned out' on the basis of their reaching a threshold score for only one of the three dimensions of the syndrome $e^{2,3}$. Such a modus operandi is inconsistent with the notion that burnout is a three-dimensional syndrome consisting of emotional exhaustion, depersonalization and reduced personal accomplishment. In a context in which burnout is nosologically uncharacterized, making claims regarding the prevalence of the syndrome is premature and potentially misleading ${ }^{3}$. Researchers should establish a clear, clinically valid (differential) diagnosis for burnout before generating prevalence estimations. Current practices in research on prevalence of physician burnout are so questionable that they call the existence of the so-called burnout epidemic into question $^{2-4}$. Interestingly, in review of the prevalence of physician burnout reported in 2018, Rosenstein et al..$^{5}$ underlined the inconclusiveness of the current state of the art in this area of research and emphasized "the importance of developing a consensus definition of burnout and of standardizing measurement tools to assess the effects of chronic occupational stress on physicians" .

Second, when listing risk factors for burnout, Murali and Banerjee ${ }^{1}$ overlooked key variables, such as personality traits. A meta-analysis conducted by Swider and Zimmerman ${ }^{6}$ revealed that the 'big five' traits of personality - neuroticism, extraversion, agreeableness, conscientiousness and openness - accounted for $21-32 \%$ of the variance in burnout scores. By comparison, in a study by Leiter and Maslach involving 6,815 individuals ${ }^{7}$, work-related factors thought to constitute the major antecedents of burnout (for example, workload, work-related reward and fairness at work) were found to explain less than $7 \%$ of the variance in burnout scores. Consistent with these findings ${ }^{6,7}$, a study relying on relative weight analysis showed that neuroticism accounted for more variance in burnout scores than work stress and social support at work ${ }^{8}$. In view of such findings, emphasizing occupational-level factors rather than general dispositional factors in descriptions of the aetiology of burnout is unwarranted.

Third, Murali and Banerjee ${ }^{1}$ affirmed that physician burnout is associated with impaired job performance, as reflected, for instance, in an increased risk of medical errors. This affirmation should be considered with caution. As pointed out by Tyssen ${ }^{9}$, a link between physician burnout and job performance has been documented in studies using subjective (self-reported) measures of performance rather than objective indicators of performance (for example, formally recorded medical errors $)^{9,10}$. More research is needed to determine the threshold at which burnout symptoms alter not only perceived but also actual functioning at work ${ }^{9}$.

Burnout has become a popular construct in occupational health research, but fundamental problems affect both the conceptualization and the measurement of the syndrome ${ }^{3,5}$. We urge researchers to, first, pay more attention to the limitations in our knowledge of burnout so as not to promulgate inaccurate and potentially misleading information and, second, define burnout at a nosological and diagnostic level. That burnout researchers remain unable to validly distinguish an individual with burnout from an individual without burnout despite $>40$ years of research on the syndrome is of great concern. Resolution of this problem should be a priority if burnout research is to progress.

There is a reply to this letter by Banerjee, $\mathrm{S}$. Nat. Rev. Clin. Oncol. https://doi.org/10.1038/ s41571-018-0151-7 (2018).

Danijela Mirkovic and Renzo Bianchi* Institute of Work and Organizational Psychology, University of Neuchâtel, Neuchâtel, NE, Switzerland. *e-mail:renzo.bianchi@unine.ch https://doi.org/10.1038/s41571-018-0150-8

1. Murali, K. \& Banerjee, S. Let's address burnout in oncologists and reimagine the way we work. Nat. Rev. Clin. Oncol. https://doi.org/10.1038/ s41571-018-0097-9 (2018).

2. Bianchi, R., Schonfeld, I. S. \& Laurent, E. Can we trust burnout research? Ann. Oncol. 28, 2320-2321 (2017).

3. Bianchi, R., Schonfeld, I. S. \& Laurent, E. Burnout: moving beyond the status quo. Int. J. Stress Manag. https://doi.org/10.1037/str0000088 (2017).

4. Schears, R. M. Defining physician burnout, and differentiating between burnout and depression - II. Mayo Clin. Proc. 92, 1455-1456 (2017).

5. Rotenstein, L. S. et al. Prevalence of burnout among physicians: a systematic review. JAMA 320 , 1131-1150 (2018).

6. Swider, B. W. \& Zimmerman, R. D. Born to burnout: a meta-analytic path model of personality, job burnout, and work outcomes. J. Vocat. Behav. 76, 487-506 (2010).

7. Leiter, M. P. \& Maslach, C. in Research in Occupational Stress and Well-being: Volume 3 Emotional and Physiological Processes and Positive Intervention Strategies (eds Perrewé, P. L. $\&$ Ganster, D. C.) 91-134 (Elsevier Science/JAI Press, NY, USA, 2004).

8. Bianchi, R. Burnout is more strongly linked to neuroticism than to work-contextualized factors. Psychiatry Res. 270, 901-905 (2018).

9. Tyssen, R. What is the level of burnout that impairs functioning? J. Intern. Med. 283, 594-596 (2018).

10. Garrouste-Orgeas, M. et al. The latroref study: medical errors are associated with symptoms of depression in ICU staff but not burnout or safety culture. Intensive Care Med. 41, 273-284 (2015).

Competing interests

The authors declare no competing interests. 